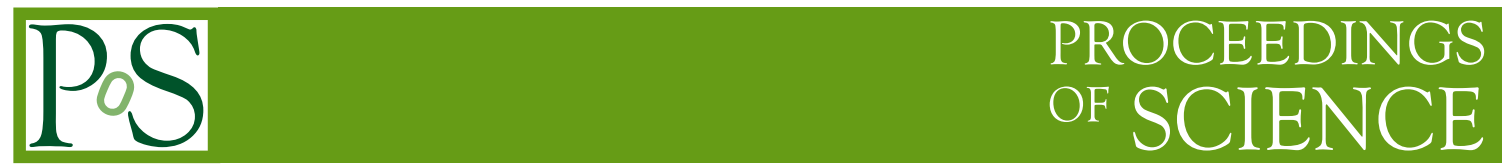

\title{
Review of recent standard model measurements
}

\author{
Paolo Azzurri ${ }^{a, *}$ \\ ${ }^{a}$ INFN sezione di Pisa, \\ Largo B. Pontecorvo, 3 - 56127 Pisa, Italy \\ on behalf of the ATLAS, CMS and LHCb collaborations \\ E-mail: Paolo.Azzurri@cern.ch
}

A review of a selection of recently released results obtained with the LHC data by the ATLAS, CMS and LHCb experiments in the domain of verifications of standard model predictions is given. Presented results range from precision measurements of fundamental parameters in inclusive processes, to differential cross section determinations and explorations of complex and rare final states.

*** The European Physical Society Conference on High Energy Physics (EPS-HEP2021), *** *** 26-30 July $2021 * * *$

*** Online conference, jointly organized by Universität Hamburg and the research center DESY ***

\footnotetext{
${ }^{*}$ Speaker
} 


\section{Introduction}

A few years after the end of the LHC Run 2 data taking, a large amount of measurements based on the Run 2 data are being released by the experiments, shifting the focus from new physics searches to more stringent and comprehensive tests of the standard model. This review contains a selection of new results presented at the EPS-HEP2021 conference, and is organised in four main parts. Section 2 reports on results with the highest precision of key parameters, Section 3 on comprehensive differential measurements of QCD processes, Section 4 includes studies with new physics interpretations, and Section 5 focuses on searches and studies of the rarest processes.

\section{High precision measurements of key parameters}

\subsection{The $\mathrm{W}$ boson mass}

The LHCb experiment performed a first measurement of the $\mathrm{W}$ boson mass with $1.7 \mathrm{fb}^{-1}$ of proton collision data at $\sqrt{s}=13 \mathrm{TeV}$ [1]. The measurement makes use of the $q / p_{\mathrm{T}}$ distribution of isolated single muons from W boson decays, shown in Fig. 1 (left), that is fitted simultaneously with the $\phi^{*}$ distribution from $\mathrm{Z}$ boson decays. The result is

$$
m_{\mathrm{W}}=80354 \pm 23 \text { (stat) } \pm 10(\exp ) \pm 17 \text { (theory) } \pm 9(\mathrm{PDF}) \mathrm{MeV}
$$

in good agreement with other direct measurements, and with indirect predictions, as shown in Fig. 1 (right). This measurement is of particular interest due to the complementary rapidity coverage of the $\mathrm{W}$ boson decay with respect to other measurements at hadron colliders. The obtained precision is limited by the statistical component, such that making use of the full LHCb available and future data will allow to improve the result.
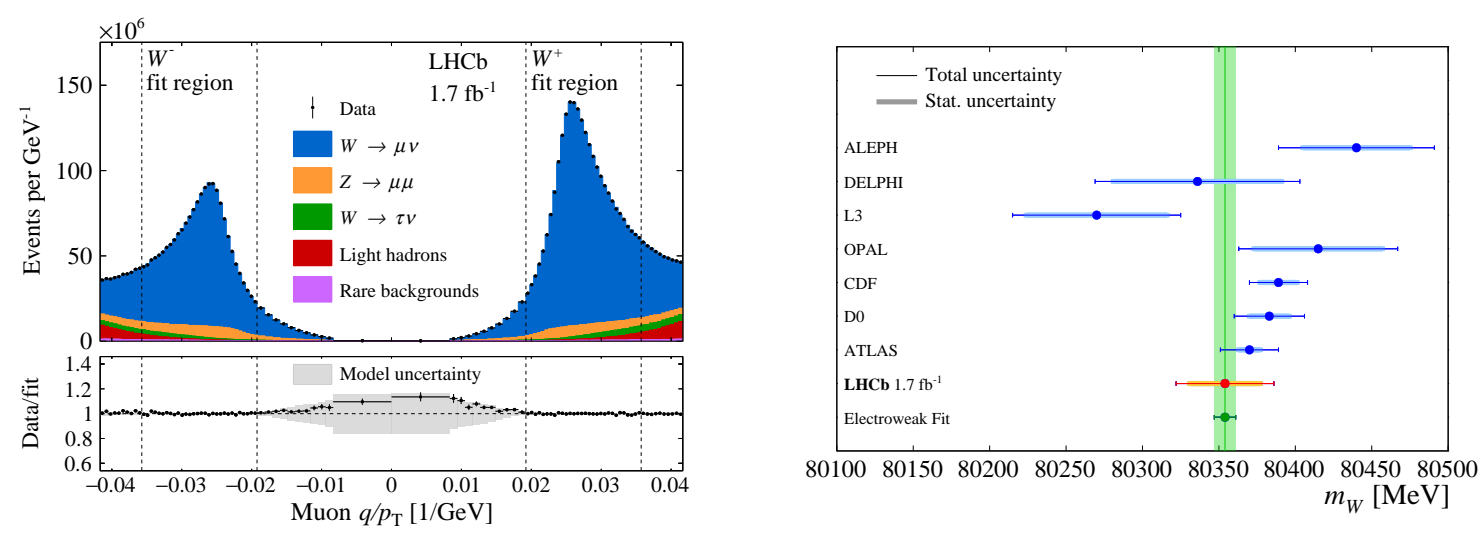

Figure 1: (left) Distribution of $q / p_{\mathrm{T}}$, compared to the model, after the fit for $m_{\mathrm{W}}$ from LHCb data [1]. (right) Comparison of $m_{\mathrm{W}}$ measurements from LEP, Tevatron and LHC data [1].

\subsection{The $\mathrm{Z}$ boson invisible width}

A first and direct measurement of the $\mathrm{Z}$ boson decay rate to invisible particles in hadron collisions has been performed by the CMS experiment using a simultaneous fit to data regions 


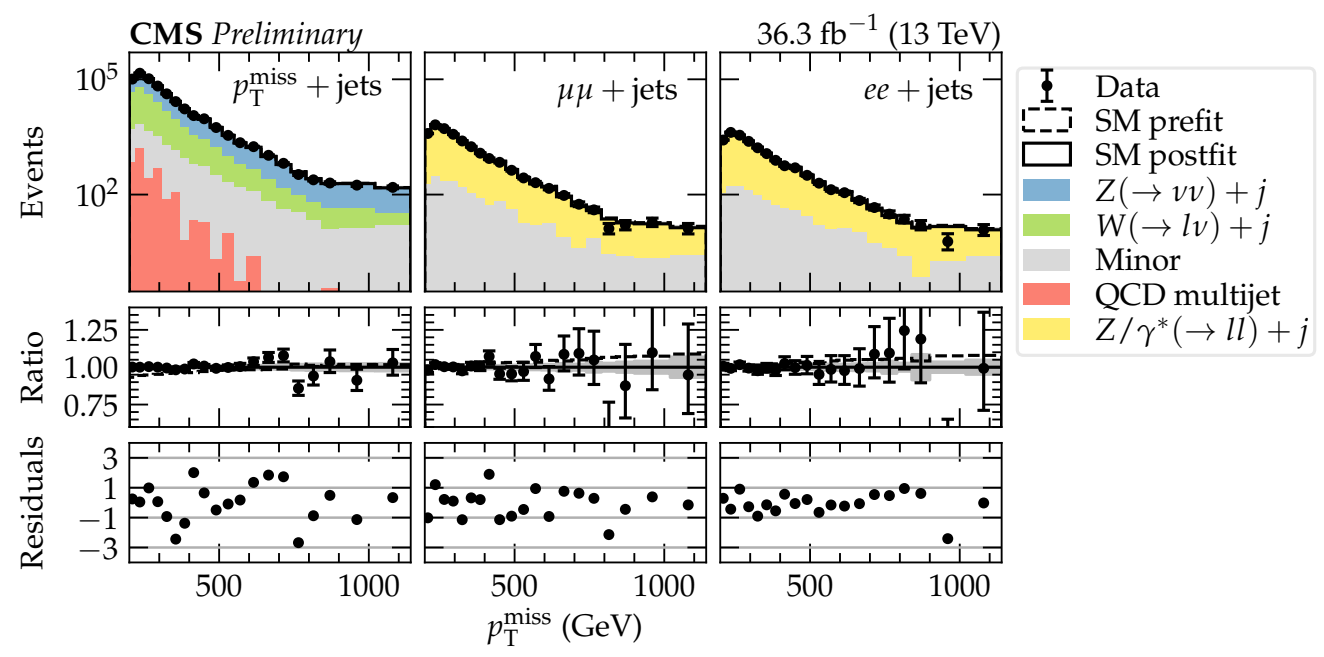

Figure 2: Postfit distribution of recoil $p_{\mathrm{T}}$ for the $p_{\mathrm{T}}^{\text {miss }}$ plus jets and the $\ell \ell$ plus jets regions. Charged leptons do not contribute to the recoil $p_{\mathrm{T}}$. Residuals are the difference between the data and the postfit results, normalised by their uncertainties [2].

containing missing transverse energy, and regions with electron and muon pairs [2]. Figure 2 shows the measured $\mathrm{Z}$ boson transverse recoil distributions used in the fit, where other dedicated data regions are employed to estimate the contribution of $\mathrm{W}$ plus jets and QCD backgrounds. The result $\Gamma_{\mathrm{Z}}^{\text {inv }}=523 \pm 3$ (stat) \pm 16 (syst) $\mathrm{MeV}$ has a precision that is limited by systematic uncertainties on lepton efficiencies and jet energy scale, and is comparable with LEP direct measurements with single photons.

\subsection{The top quark mass interpretation}

A relation between the top quark mass parameter in simulation and the MSR field-theoretical mass scheme has been obtained by ATLAS with the jet mass distribution of large-radius jets containing boosted hadronic top quark decays [3]. The relation is found to be $m_{t}^{\mathrm{MC}}-m_{t}^{\mathrm{MSR}}(1 \mathrm{GeV})=$ $80_{-400}^{+350} \mathrm{MeV}$, with major uncertainty contributions from the theory predictions, the fit methodology and the underlying event modelling. The result is a further step to understand and reduce uncertainties on direct top quark mass measurements, and towards a universal interpretation of the top quark mass.

\section{Differential tests of predictions}

\subsection{Inclusive and multiple jets}

A first measurement of inclusive jet cross sections in proton collisions at $5 \mathrm{TeV}$ has been performed by the CMS experiment, differentially in transverse momentum and rapidity [4]. Results shown in Fig. 3 (left) are compared with QCD predictions and can complement data from other collision energies in offering new constraints to proton PDFs. New results on inclusive jet productions have been also provided by CMS with $13 \mathrm{TeV}$ proton collision data [5], including 

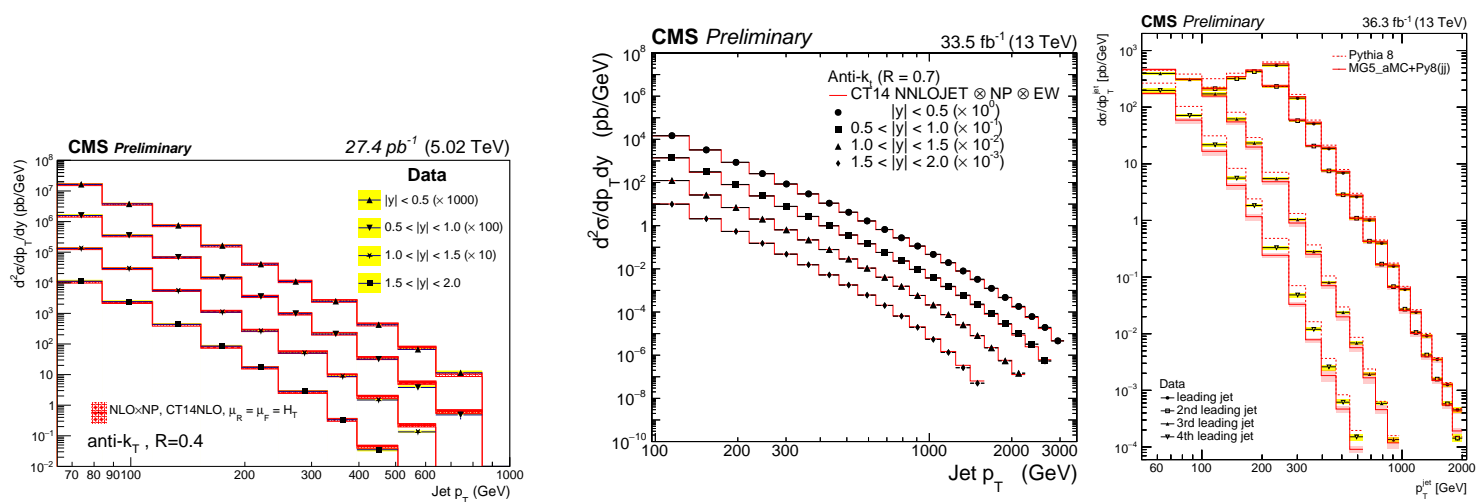

Figure 3: Inclusive jet cross sections as a function of particle level jet $p_{\mathrm{T}}$ in diferent rapidity regions with (left) jet $R=0.4$ at $5 \mathrm{TeV}$ [4] and (center) with jet $R=0.7$ at $13 \mathrm{TeV}$ [5]. Data results are compared to perturbative QCD predictions. (right) Differential cross sections as a function of the event $p_{\mathrm{T}}$-leading jets. The yellow band represents the total data experimental uncertainty and is compared to different predictions [6].

double-differential results with different jet radii, as shown in Fig. 3 (center). These measurements are combined with multi-differential top quark pair cross sections in a comprehensive QCD analysis to extract proton PDFs, the strong coupling, and the top quark mass that is determined to be $m_{t}^{\text {pole }}=170.2 \pm 0.6($ fit $) \pm 0.1$ (model) $\mathrm{GeV}$.

Another recent CMS analysis explores the properties of multijet events, measuring their multiplicity, $p_{\mathrm{T}}$ distributions, shown in Fig. 3 (right), and their azimuthal correlations [6]. Data results are compared to predictions using NLO QCD matrix-element calculations combined with conventional parton shower as well as with Parton Branching (PB) Transverse Momentum Dependent (TMD) parton densities and PB-TMD initial state parton shower.

\section{2 $\mathrm{Z}$ bosons in association with jets}

A comprehensive study of the production of $\mathrm{Z}$ bosons in association with jets in proton collisions at $13 \mathrm{TeV}$ has been performed by CMS [7], including a large number of single- and double-differential observables unfolded to particle level, as shown in Fig. 4 (left), and compared with predictions from several Monte Carlo event generators, as well as with expectations at different precision orders in perturbative QCD. A dedicated study of azimuthal correlations in Z plus jets events [8] focuses on the $p_{\mathrm{T}}$-evolution of the correlations, as the one shown in Fig. 4 (center), and indicates the shortcomings of predictions in describing the data in regions where different production contributions become relevant.

\section{Z plus high $p_{\mathrm{T}}$ jets}

Proton collisions at $13 \mathrm{TeV}$ have been used by ATLAS to measure properties of events with a $\mathrm{Z}$ boson and at least one high- $p_{\mathrm{T}}$ jet [9]. Focus is brought into investigating $\mathrm{Z}$ plus jet back-to-back and collinear productions, classified by the angular distance shown in Fig. 4 (right). Data results show a fair agreement with state-of-the-art Monte Carlo predictions, and larger discrepancies with less precise models. 

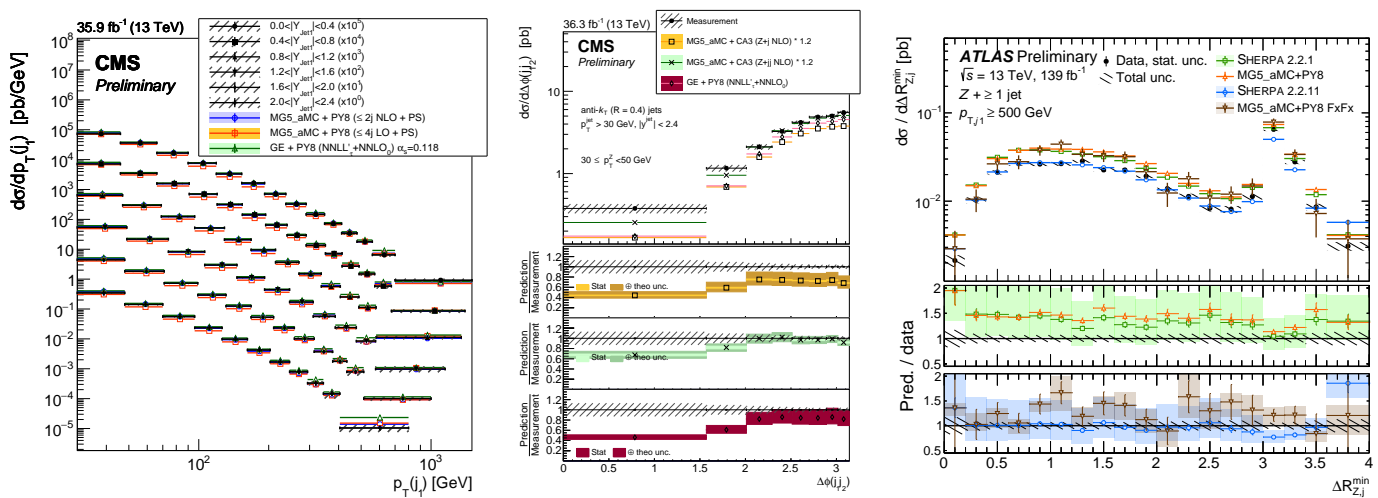

Figure 4: $\mathrm{Z}$ plus jet measurements. (left) Double differential cross sections as a function of leading jet $p_{\mathrm{T}}$ and $|y|$, compared to predictions [7]. (center) Cross sections as a function of dijet azimuthal opening angle in a given $\mathrm{Z}$ boson $p_{\mathrm{T}}$-range, compared to predictions from different models [8]. (right) Differential cross section as a function of angular distance between the $\mathrm{Z}$ and the closest jet, for events with high- $p_{\mathrm{T}}$ jets [9].

\section{Z plus bottom jets}

Integrated and differential measurements of the associated production of a $\mathrm{Z}$ boson and at least one or two $b$ jets are reported by CMS in $13 \mathrm{TeV}$ proton collision data [10]. Figure 5 (left) shows the distribution of $\mathrm{Z}$ plus two $\mathrm{b}$ jets as a function of the $\mathrm{b}$-jets angular separation. Results are compared with different predictions and while predictions with higher-order QCD calculations tend to describe the differential shapes of the data better, they also seem to overestimate the data overall normalization.
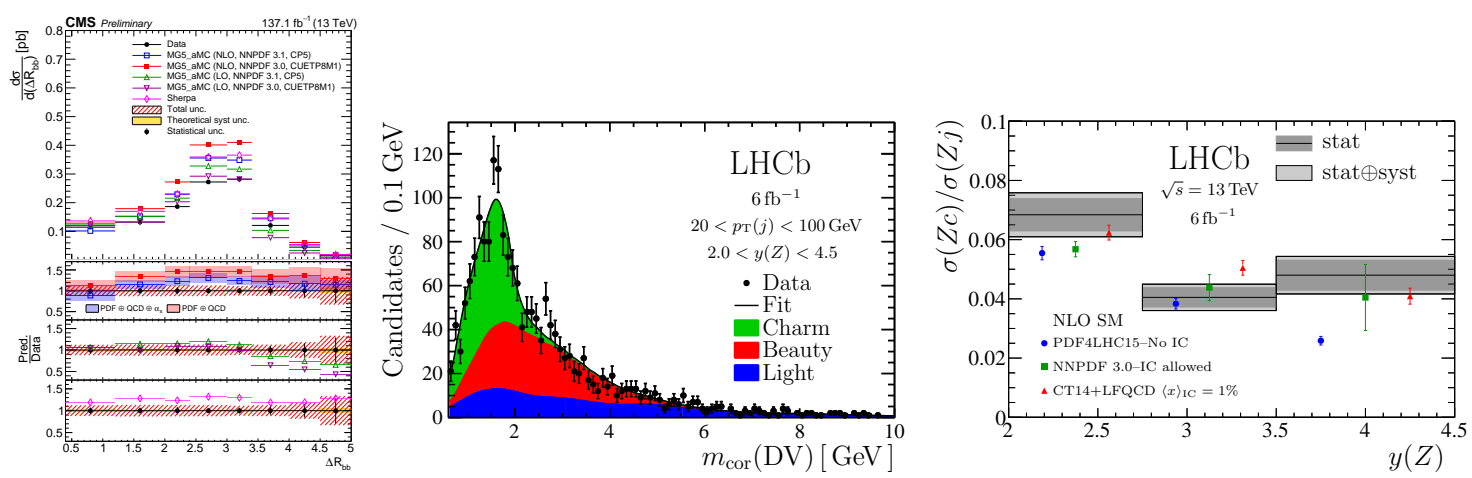

Figure 5: (left) Differential cross section as a function of the angular separation between two b jets in $\mathrm{Z}$ plus bb events [10]. (center) Distribution of displaced vertex mass in $Z$ plus jets events [11]. (right) Measured ratio of charm production for three intervals of $Z$ rapidity, compared to different NLO predictions [11].

\section{Z plus charm jets}

The rate of charm jets produced in association with a $\mathrm{Z}$ boson in the forward region has been measured by the LHCb experiment with $6 \mathrm{fb}^{-1}$ of proton collision data at $13 \mathrm{TeV}$ [11]. The displaced vertex mass, shown in Fig. 5 (center), and track multiplicity are used to distinguish the jet flavour components. The measurement is performed in intervals of $\mathrm{Z}$ boson rapidity, and compared 
to NLO QCD predictions, as shown in Fig. 5 (right). The results in the largest rapidity region $(3.5<y(Z)<4.5)$ seem to prefer predictions that include intrinsic charm in the proton PDFs.

\subsection{Photon pairs}

Events with two photons have been extensively studied and measured by ATLAS, including fiducial and differential cross sections [12]. Good agreement is generally found with the predictions when taking into account higher-order terms in perturbative QCD. The region of low diphoton invariant mass is sensitive to multi-jet configurations, which are modelled with limited precision in all predictions.

\section{Measurements with searches for new physics effects}

\subsection{Single top quark polarisation}

The three components of the single top quark polarisation produced in the $t$-channel have been measured by ATLAS by fitting the rates of lepton angular observables [13]. The results, shown in Fig. 6 (left) for two components, are in agreement with NLO QCD predictions. The measured angular differential cross sections are also fitted to extract limits on the real and imaginary part of the $O_{t W}$ effective field theory (EFT) operator, that are found to be consistent with standard model predictions and improving previous constraints.
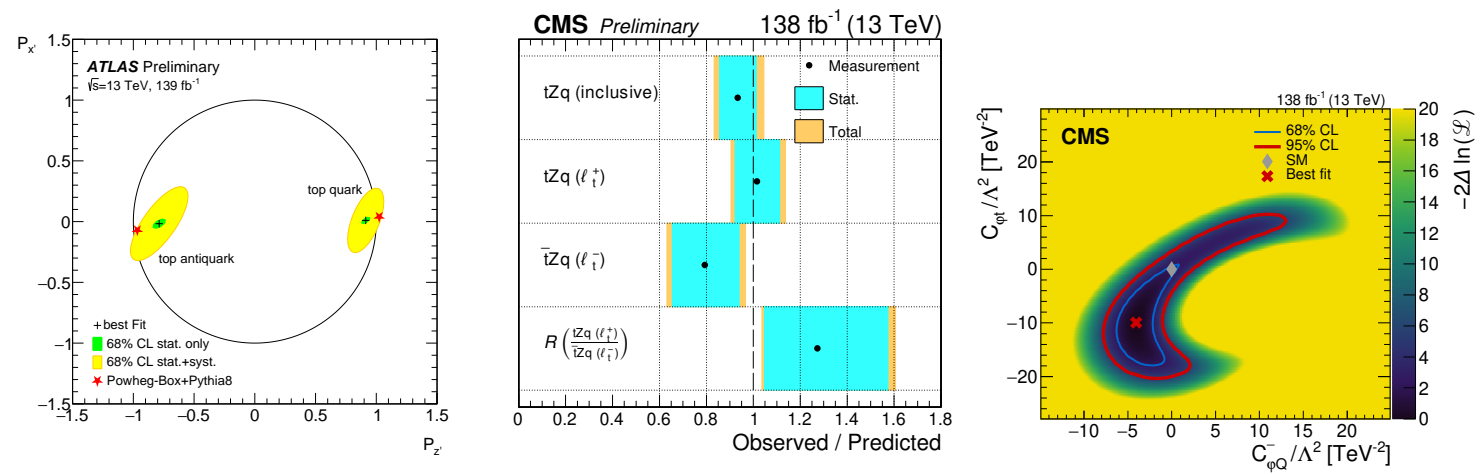

Figure 6: (left) Single top polarisation measurements in the two-dimensional $\left(x^{\prime}, z^{\prime}\right)$ polarisation parameter space [13]. (center) Signal strengths of the tZq cross section measured inclusively, and separately for the top quark and antiquark cross sections, and their ratio [14]. (right) Two-dimensional likelihood of the Wilson coefficients $c_{\phi Q}^{-}$and $c_{\phi t}[15]$.

\subsection{Single and double top quarks in association with a $\mathrm{Z}$ boson}

Inclusive and differential cross section measurements of single top quark production in association with a $\mathrm{Z}$ boson in proton collisions at $13 \mathrm{TeV}$ have been determined by CMS making use of events with three leptons and at least one b-jet [14]. The inclusive cross section is measured at $\sim 10 \%$ precision, and top quark and antiquark production cross sections are established separately, together with their ratio, as shown in Fig. 6 (center). All results are in good agreement with predictions, including the measured top quark spin asymmetry. 
Multilepton final states with at least one b-jet have also been investigated by CMS to search for new top quark interactions in the production of either one or two top quarks with a $\mathrm{Z}$ boson [15]. A multivariate analysis relying upon machine-learning techniques is used to enhance the sensitivity to effects arising from five possible EFT operators, and confidence intervals are determined for the corresponding coefficients, such as the ones shown in Fig. 6 (right). All results are consistent with standard model predictions at $95 \%$ confidence level.

\subsection{High- $p_{\mathrm{T}}$ top quarks}

Dedicated studies of the production of top quark pairs with a large- $p_{\mathrm{T}}$ hadronic decay have been performed by ATLAS [16]. The measured $p_{\mathrm{T}}$-differential cross section is shown in Fig. 7 (left) together with predictions from different models and calculations. Results include several fiducial and differential cross sections, and the derivation of constraints on the $c_{t G}$ and $c_{t q}^{(8)}$ EFT coefficients, that are in agreement with standard model expectations.
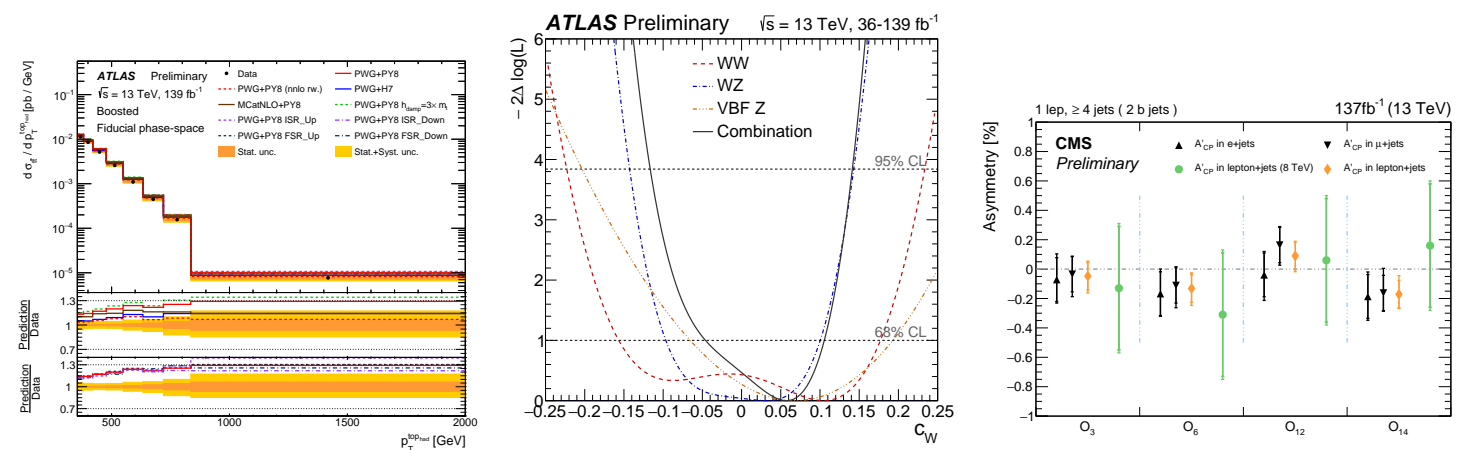

Figure 7: (left) Top pairs differential cross section relative to the hadronic top decay $p_{\mathrm{T}}[16]$. (center) Likelihood profile of the Wilson coefficient $c_{W}$ as obtained from individual measurements and their combination [17]. (right) Effective CP asymmetries from different observables of semileptonic top pair decays [18].

\subsection{Combined effective field theory fits}

The ATLAS Collaboration performed a combined fit of diboson and vector boson fusion differential cross section measurements to constrain the coefficients of different EFT operators [17]. Figure 7 (center) shows an example of the individual and combined constraints on $c_{W}$. The work highlights how combined interpretations of multiple measurements can be carried out towards global EFT interpretations.

\subsection{CP violation in double top quark events}

A search for $\mathrm{CP}$ violation in the production and decay of top quark pairs using the semileptonic decay channel has been performed by CMS [18]. Four CP-violating asymmetries, are defined from observables built from the final state four-vectors, and measured with template fits. Results are shown in Fig. 7 (right), and exhibit no evidence for CP-violating effects, improving the precision of previous results by an important factor. 


\section{Exploration of rare processes}

\subsection{Production of four top quarks}

The production of four top-quarks in proton collisions at $13 \mathrm{TeV}$ has been measured by ATLAS in events that contain a single lepton or an opposite-sign lepton pair, in association with multiple jets [19]. Results are combined with a previous measurement performed in the multilepton final state, yielding a combined signal strength $\mu=2.0_{-0.6}^{+0.8}$ with an observed significance of 4.7 standard deviations, and a consistency to the standard model prediction within 2.0 standard deviations.

\subsection{Production of three $W$ bosons}

The first observation of triple $\mathrm{W}$ boson production has been reported by the ATLAS experiment, making use of events with three leptons, and of events with two same-sign leptons and two jets [20]. Boosted decision trees, shown in Fig. 8 (left) for the three lepton category, are used to improve the separation between signal and backgrounds. The results yield a signal strength $\mu=1.66 \pm 0.28$ with an observed (expected) signal significance of 8.2 (5.4) standard deviations.
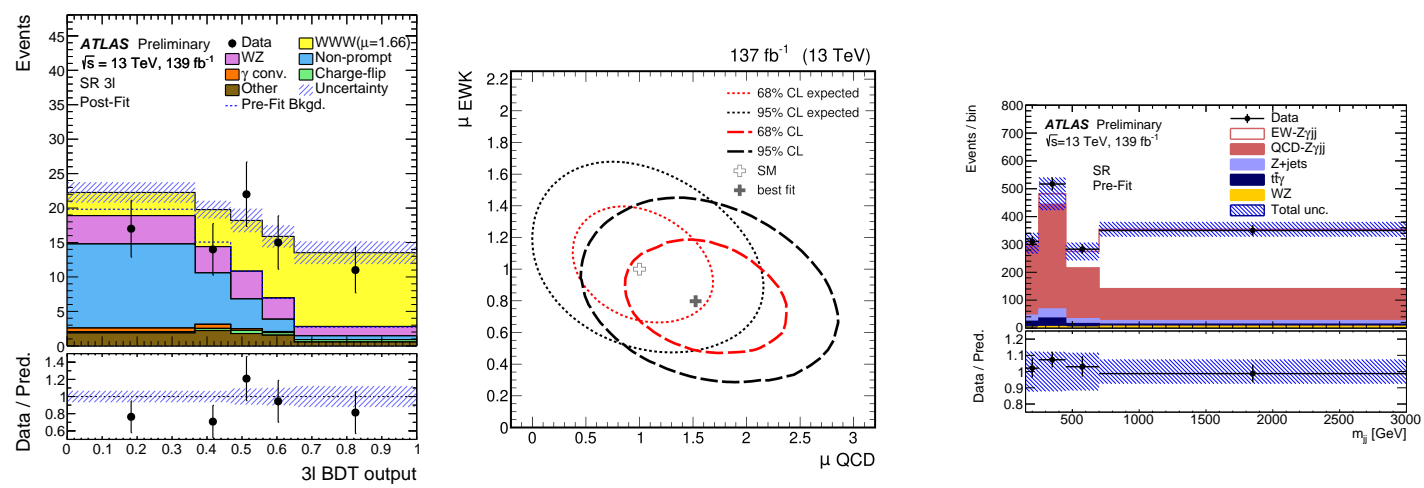

Figure 8: (left) BDT distribution of the three lepton category for the measurement of WWW production [20]. (center) Expected and observed CL contours for the EW and QCD semileptonic WV plus two jets signal strengths [21]. (right) Dijet invariant mass distribution for the measurement of the electroweak production of a $\mathrm{Z} \gamma$ pair in association with two jets [22].

\subsection{Semileptonic vector boson scattering}

The first evidence of vector boson scattering in the semileptonic channel has been reported by CMS analysing events with one lepton, missing transverse momentum, a dijet system with large invariant mass, and an additional hadronic signature consistent with the decay of a W/Z boson [21]. The measured signal strength is $\mu=0.85_{-0.20}^{+0.24}$ with an observed (expected) signal significance of 4.4 (5.1) standard deviations. Figure 8 (center) shows the result of a two-dimensional fit of the EW and QCD WV production components, showing an overall consistency with the standard model predictions.

\subsection{Electroweak production of two jets in association with a photon and a $\mathrm{Z}$ boson}

The electroweak production of a $\mathrm{Z} \gamma$ pair in association with two jets has been measured by the ATLAS experiment both in the channel with two leptons [22], and in the channel with missing 
transverse momentum [23]. Figure 8 (right) shows the dijet invariant mass distribution that is fitted in the two lepton channel, yielding a signal strength of $\mu=0.95_{-0.13}^{+0.14}$. In the missing transverse momentum channel the fitted signal strength is measured to be $\mu=1.03 \pm 0.25$, reaching an observed (expected) signal significance of 5.2 (5.1) standard deviations.

\subsection{Search for flavour-changing neutral current interactions of the top quark and the Higgs boson}

The CMS experiment searched for flavour-changing neutral current interactions of top quarks and the Higgs boson, in final states with a top quark and a Higgs boson decaying alternatively to photon pairs [24], and to b-jet pairs [25]. In both channels multivariate techniques are employed to separate the searched signal signatures from backgrounds. No significant excess above the background predictions is observed, and 95\% confidence level upper limits are derived from the photon (b-jet) channels, on the branching fractions $B(t \rightarrow \mathrm{H} u)<1.9(7.9) 10^{-4}$ and $B(t \rightarrow \mathrm{H} c)<$ 7.3(9.4) $10^{-4}$.

\subsection{Production of three $\mathrm{J} / \psi$ mesons}

The CMS experiment reported the first observation of the triple $\mathrm{J} / \psi$ meson production [26]. Making use of a fit of dimuon mass distributions, five events are found to be consistent with triple $\mathrm{J} / \psi$ production, and one event with combinatorial background. A fiducial cross section is derived to be $\sigma(\mathrm{pp} \rightarrow \mathrm{J} / \psi \mathrm{J} / \psi \mathrm{J} / \psi \mathrm{X})=272_{-104}^{+141}$ (stat.) \pm 17 (syst.) fb and compared to theoretical expectations. Under the assumption of factorisation of multiple hard scattering probabilities an effective DPS cross section of $\sigma_{\mathrm{DPS}}^{\text {eff }}=2.7_{-1.0}^{+1.4}(\exp )_{-1.0}^{+1.5}$ (theo) $\mathrm{mb}$ is derived, that is consistent with values from double-quarkonium measurements, but significantly smaller than the effective DPS values derived from other double-particle final states including jets, photons, and electroweak bosons.

\section{Summary}

The new results released for the EPS-HEP2021 conference by the LHC experiments in the domain of studies of the standard model are impressive both in terms of quantity and relevance. These include the first precision measurement of the W boson mass with forward decays by LHCb, and the first precision measurement of the $\mathrm{Z}$ invisible decay rates by CMS, that matches the precision of direct determinations from LEP data. The reported observation of triple-W production by ATLAS, and evidence of VBS processes in the semileptonic final state by CMS represent important milestones in multiboson physics. Other remarkable results include measurements of four top-quark productions by ATLAS and of associated top-quark and Z boson productions by CMS, the observation of triple $\mathrm{J} / \psi$ production by CMS, and measurements of $\mathrm{Z} \gamma \mathrm{VBS}$ with invisible final states by ATLAS. A large number of multi-differential measurements in multijet and Z plus jets final states have been produced by all experiments, including an interesting determination of $\mathrm{Z}$ plus charm in the forward region by LHCb that hints to the presence of a proton intrinsic charm component. More explorations and verifications of standard model predictions are still to be performed both with existing Run 2 data, and with the upcoming Run 3. This is a very important time for such measurements with the LHC data, to consolidate our knowledge of possible processes 
in proton-proton interactions, and possibly reveal indications of new effects that can lead us to the next discoveries.

\section{References}

[1] R. Aaij et al. LHCb Collaboration, Measurement of the W boson mass, [arXiv:2109.01113 [hep-ex]].

[2] CMS Collaboration, Precision measurement of the $Z$ invisible width with the CMS experiment in pp collisions at $\sqrt{s}=13$ TeV CMS-PAS-SMP-18-014.

[3] ATLAS Collaboration, A precise interpretation for the top quark mass parameter in ATLAS Monte Carlo simulation, ATL-PHYS-PUB-2021-034.

[4] CMS Collaboration, Measurement of the double-differential inclusive jet cross section in proton-proton collisions at $\sqrt{s}=5.02 \mathrm{TeV}$, CMS-PAS-SMP-21-009.

[5] CMS Collaboration, Measurement and QCD analysis of double-differential inclusive jet cross sections in p collisions at $\sqrt{s}=13 \mathrm{TeV}$, CMS-PAS-SMP-20-011.

[6] CMS Collaboration, Cross section measurements of jet multiplicity and jet transverse momenta in multijet events at $\sqrt{s}=13 \mathrm{TeV}$, CMS-PAS-SMP-21-006.

[7] CMS Collaboration, Measurement of multi-differential cross sections for the production of a $Z$ boson in association with jets in proton-proton collisions at $\sqrt{s}=13 \mathrm{TeV}$, CMS-PAS-SMP19-009.

[8] CMS Collaboration, Azimuthal correlations in Z+jets events at $13 \mathrm{TeV}$, CMS-PAS-SMP-21003.

[9] ATLAS Collaboration, Measurements of the production cross section of a $Z$ boson in association with high transverse momentum jets in pp collisions at $\sqrt{s}=13 \sim \mathrm{TeV}$ with the ATLAS detector, ATLAS-CONF-2021-033.

[10] CMS Collaboration, Measurement of $Z+b$ jets cross section in proton-proton collisions at $\sqrt{s}=13 \mathrm{TeV}$, CMS-PAS-SMP-20-015.

[11] R. Aaij et al. LHCb Collaboration, Study of $Z$ bosons produced in association with charm in the forward region, [arXiv:2109.08084 [hep-ex]].

[12] ATLAS Collaboration, Measurement of the production cross section of pairs of isolated photons in pp collisions at $13 \mathrm{TeV}$ with the ATLAS detector, [arXiv:2107.09330 [hep-ex]].

[13] ATLAS Collaboration, Measurement of the polarisation of single top quarks and antiquarks produced in the t-channel collected with the ATLAS detector at $\sqrt{s}=13 \mathrm{TeV}$ and bounds on the tWb dipole operator, ATLAS-CONF-2021-027. 
[14] CMS Collaboration, Inclusive and differential cross section measurements of single top quark production in association with a Z boson in proton-proton collisions at $\sqrt{s}=13 \mathrm{TeV}$, CMSPAS-TOP-20-010.

[15] A. Tumasyan et al. CMS Collaboration, Probing effective field theory operators in the associated production of top quarks with a Z boson in multilepton final states at $\sqrt{s}=13 \mathrm{TeV}$, [arXiv:2107.13896 [hep-ex]].

[16] ATLAS Collaboration, Measurements of differential cross-sections in top-quark pair events with a high transverse momentum top quark and limits on beyond the Standard Model contributions to top quark pair production with the ATLAS detector, ATLAS-CONF-2021-031.

[17] ATLAS Collaboration, Combined effective field theory interpretation of differential crosssections measurements of $W W, W Z, 4 \ell$, and Z-plus-two-jets production using ATLAS data, ATL-PHYS-PUB-2021-022.

[18] CMS Collaboration, Search for CP violation in top quark pair events in the lepton+jets channel at $\sqrt{s}=13 \mathrm{TeV}$, CMS-PAS-TOP-20-005.

[19] ATLAS Collaboration, Measurement of the t $\bar{t} t \bar{t}$ production cross section in p p collisions at $\sqrt{s}=13 \mathrm{TeV}$ with the ATLAS detector, [arXiv:2106.11683 [hep-ex]].

[20] ATLAS Collaboration, Observation of $W W W$ production in pp collisions at $\sqrt{s}=13$ TeV with the ATLAS detector, ATLAS-CONF-2021-039.

[21] CMS Collaboration, Search for vector boson scattering at the LHC Run 2 with CMS data in the semi-leptonic lvqq final state, CMS-PAS-SMP-20-013.

[22] ATLAS Collaboration, Measurement of the cross-section of the electroweak production of a $Z \gamma$ pair in association with two jets in p collisions at $\sqrt{s}=13 \mathrm{TeV}$ with the ATLAS detector, ATLAS-CONF-2021-038.

[23] ATLAS Collaboration, Observation of electroweak production of two jets in association with an isolated photon and missing transverse momentum, and search for a Higgs boson decaying into invisible particles at $13 \mathrm{TeV}$ with the ATLAS detector, [arXiv:2109.00925 [hep-ex]].

[24] CMS Collaboration, Search for flavor-changing neutral current interactions of the top quark and the Higgs boson in the diphoton decay channel in proton-proton collisions at $\sqrt{s}=13 \mathrm{TeV}$, CMS-PAS-TOP-20-007.

[25] CMS Collaboration, Search for flavor-changing neutral current interactions of the top quark and the Higgs boson decaying to a bottom quark-antiquark pair at $\sqrt{s}=13 \mathrm{TeV}$, CMS-PASTOP-19-002.

[26] CMS Collaboration, Observation of triple $\mathrm{J} / \psi$ meson production in proton-proton collisions at $\sqrt{s}=13 \mathrm{TeV}$, CMS-PAS-BPH-21-004. 